NIST Technical Note 2139

\title{
A Perspective on Forest Fuel Bed Ignition by Firebrands
}

Jiann C. Yang

This publication is available free of charge from:

https://doi.org/10.6028/NIST.TN.2139

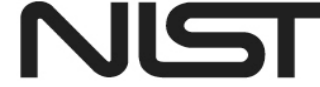

National Institute of Standards and Technology U.S. Department of Commerce 
NIST Technical Note 2139

\section{A Perspective on Forest Fuel Bed Ignition by Firebrands}

Jiann C. Yang

Fire Research Division Engineering Laboratory

This publication is available free of charge from:

https://doi.org/10.6028/NIST.TN.2139

February 2021

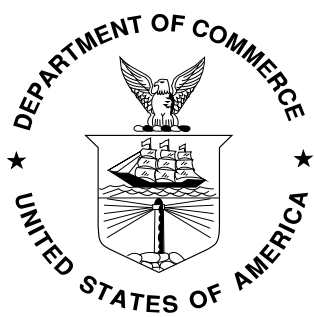

U.S. Department of Commerce

Wynn Coggins, Acting Secretary

National Institute of Standards and Technology James K. Olthoff, Performing the Non-Exclusive Functions and Duties of the Under Secretary of Commerce for Standards and Technology \& Director, National Institute of Standards and Technology 
Certain commercial entities, equipment, or materials may be identified in this document in order to describe an experimental procedure or concept adequately. Such identification is not intended to imply recommendation or endorsement by the National Institute of Standards and Technology, nor is it intended to imply that the entities, materials, or equipment are necessarily the best available for the purpose.

National Institute of Standards and Technology Technical Note 2139

Natl. Inst. Stand. Technol. Tech. Note 2139, 9 pages (February 2021)

CODEN: NTNOEF

This publication is available free of charge from:

https://doi.org/10.6028/NIST.TN.2139 


\begin{abstract}
A different perspective on forest fuel bed ignition by firebrands is presented to obtain a correlation between the ignition time of a fuel bed by a glowing firebrand and fuel moisture content. A firebrand heating efficiency akin to spray cooling efficiency was introduced and used in the analysis. The idea of using a firebrand heating efficiency of a forest fuel bed coupled with proper dimensional analysis may offer an effective way to correlate prospective experimental fuel bed ignition data as function of fuel properties, fuel configuration, firebrand shower characteristics, and wind.
\end{abstract}

\title{
Keywords
}

Firebrand; forest fuel; ignition; moisture content. 


\section{Table of Contents}

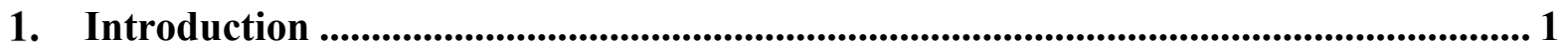

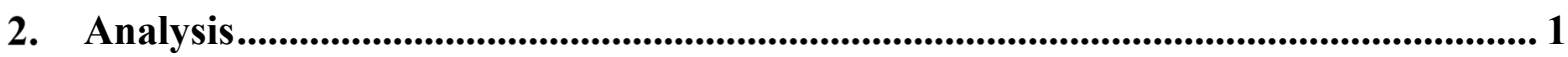

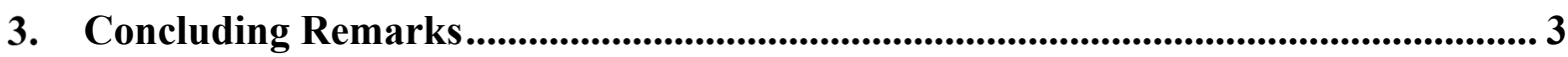

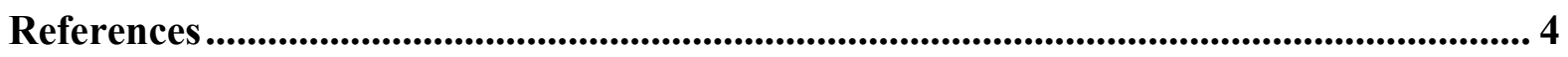

\section{List of Figures}

Fig. 1. Illustration of firebrand ignition of semi-infinite (thermally thick) fuel bed. .............. 2 


\section{Introduction}

The ignition of forest fuel bed by firebrands has been a subject of great interest due to its relevance to spot ignition and forest fire spread. The propensity for ignition of the forest fuel bed depends on many parameters, including thermophysical properties of the fuel, fuel loading density, fuel configuration, ambient conditions, firebrand mass and number fluxes onto fuel bed, contact area and time between firebrands and fuel bed, and fuel moisture content.

A correlation between ignition time and moisture content for pine needles attacked by firebrands under wind condition was published by Yin et al. [1]. The correlation was developed using detailed theoretical consideration of the governing heat transfer processes that occurred when a glowing firebrand heated and a subsequent ignition of a thermally thick fuel bed. Based on their analysis, a relationship was found, $\sqrt{t_{i g}} \sim M C$ was found, where $t_{i g}$ is the ignition time of the fuel bed and $M C$ is the moisture content of the fuel. In this note, a different perspective is provided to show that the same relationship between $t_{i g}$ and $M C$ could be easily obtained using a simple alternative approach.

\section{Analysis}

Let us first examine the case where the fuel bed is assumed to be totally dry and treated as a semi-infinite medium (thermally thick), as illustrated in Fig. 1. Consider that the heat transfer from the glowing firebrands on the fuel bed surface can be viewed and simplified to a constant heat flux being imposed on the fuel bed surface. With constant thermophysical properties, the solution for the one-dimensional transient conduction heat transfer through the fuel bed with a constant surface heat flux as the boundary condition is [2]

$$
T(x, t)-T_{o}=\frac{2 q_{s}^{\prime \prime}(\sqrt{\alpha t / \pi})}{k} \exp \left(\frac{-x^{2}}{4 \alpha t}\right)-\frac{q_{s}^{\prime \prime} x}{k} \operatorname{erfc}\left(\frac{x}{2 \sqrt{\alpha t}}\right)
$$

where $T$ is the fuel bed temperature, $T_{o}$ is the initial fuel bed temperature, $q_{s}^{\prime \prime}$ is the constant surface heat flux, $\alpha$ is the thermal diffusivity of the fuel bed, $t$ is the time, $k$ is the fuel bed thermal conductivity, $x$ is the distance measured from the fuel bed surface $(x=0)$.

Following Yin et al. [1], if we assume the ignition time $t_{i g}$ corresponds to the time that the surface temperature reaches $T\left(x=0, t_{i g}\right)=T_{i g}$, then Eq. (1) becomes

$$
T_{i g}-T_{o}=\frac{2 q_{s}^{\prime \prime}\left(\sqrt{\alpha t_{i g} / \pi}\right)}{k}
$$

Note that the penetration depth used in Yin et al. [1] is implicitly based on the approximate integral solution of one-dimensional heat conduction in a semi-infinite medium. 

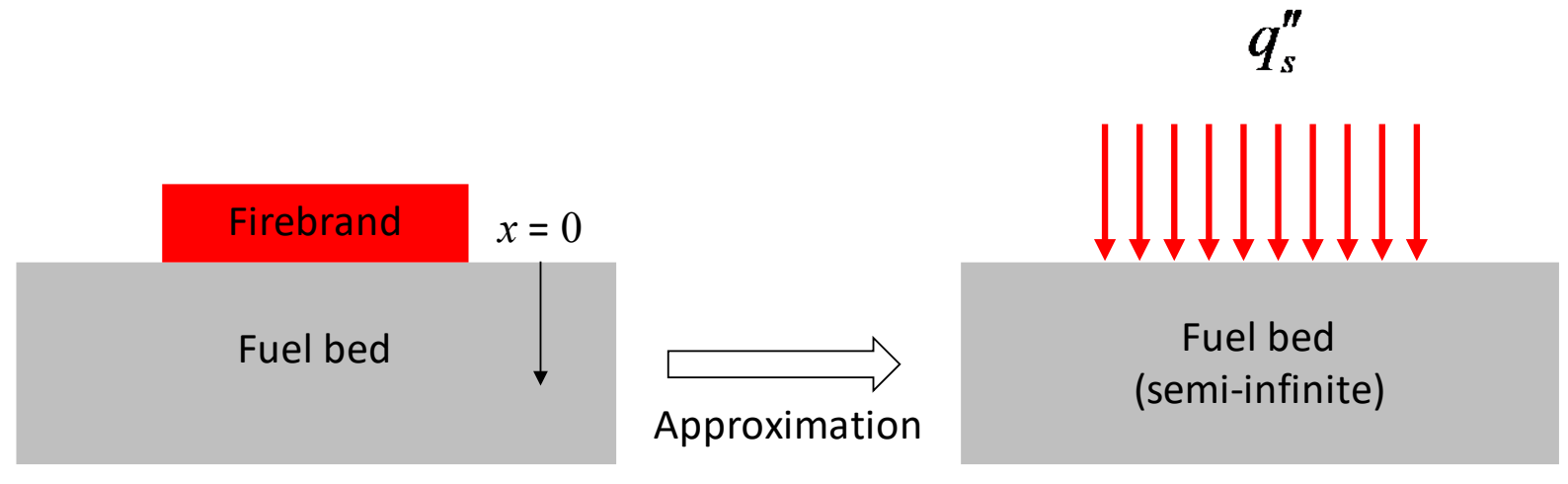

Fig. 1. Illustration of firebrand ignition of semi-infinite (thermally thick) fuel bed.

If we perceive firebrand heating of a fuel bed as a reverse problem of spray cooling of a heated surface, we could make use of the spray cooling efficiency concept ${ }^{1}$ which is commonly used in the spray cooling literature [3] and introduce a so-called firebrand heating efficiency $(\eta)$. In this context, the heating efficiency could be defined as the fraction of the net surface heat flux used to heat the fuel bed to $T_{i g}$. This accounts for the fact that in addition to heating the fuel bed part of the net surface heat flux would also be used to evaporate the moisture content of fuel bed if the fuel bed is not completely dry. Note that the radiative and convective losses from the firebrand, which were included in Yin et al. [1], could also be implicitly incorporated in $\eta$.

Then, for a moist fuel bed, $q_{s}^{\prime \prime}$ would now be replaced by $\eta q_{s}^{\prime \prime}$ in Eq. (2), which becomes

$$
T_{i g}-T_{o}=\frac{2 \eta q_{s}^{\prime \prime}\left(\sqrt{\alpha t_{i g} / \pi}\right)}{k} \quad 0<\eta \leq 1
$$

If the fuel bed is completely dry, $\eta$ is equal to 1 , and the surface heat flux would be used solely to heat up the fuel bed to $T_{i g}$. Implicit in the above discussion are the assumptions that the moisture evaporation process is not coupled with the heat transfer process within the fuel bed and that $t_{i g}$ is much shorter than the burn-out time $\left(t_{b o}\right)$ of the firebrand $\left(t_{i g}<<t_{b o}\right)$.

These two assumptions were also implied in the analysis by Yin et al. [1]. In addition, the assumption of constant heat flux at the fuel bed surface is reasonable if $t_{i g}<<t_{b o}$ for thermally thick fuel bed. Since one would expect more thermal energy (higher $\eta$ ) is available to heat the fuel with lower $M C$, it is reasonable as a first-order approximation to assume $\eta$ to be linearly inversely proportional to $M C$.

$$
\eta \sim \frac{1}{M C}
$$

\footnotetext{
${ }^{1}$ The spray cooling efficiency is defined as the ratio of the actual heat transfer due to the effect of the impinging droplets to the total heat transfer which is required for the complete evaporation of the droplets.
} 
It is interesting to note that if one were to apply the definition of $\eta$ to the resulting equations in Yin et al. [1], one could also arrive at Eq. (4) formally.

For a given $T_{i g}$ and a fuel bed with given initial conditions ( $T_{o}$ and $\left.q_{s}^{\prime \prime}\right)$ with known thermophysical properties ( $\alpha$ and $k$ ), one could easily arrive at the same correlation given by Yin et al. [1] from Eq. (3)

$$
\sqrt{t_{i g}} \sim M C
$$

The above concept could be extended to a thermally thin fuel bed. In this case, the temperature of the fuel bed is assumed to be spatially uniform but time varying. Under this condition, the governing equation for a completely dry fuel bed with a thickness $\delta$ simply becomes

$$
\rho V c_{p} \frac{d T}{d t}=q_{s}^{\prime \prime} A
$$

where $\rho, c_{p}, A$, and $V(=A \delta)$ are the fuel density, fuel heat capacity, fuel surface area exposed to the imposed surface heat flux, and fuel volume respectively. Integrating Eq. (6),

$$
T_{i g}-T_{o}=\frac{q_{s}^{\prime \prime}}{\rho \delta c_{p}} t_{i g}
$$

Following the same discussion on thermally thick fuel bed given above, for a moist fuel bed, $q_{s}^{\prime \prime}$ would now be replaced by $\eta q_{s}^{\prime \prime}$ in Eq. (7), which becomes

$$
T_{i g}-T_{o}=\frac{\eta q_{s}^{\prime \prime}}{\rho \delta c_{p}} t_{i g} \quad 0<\eta \leq 1
$$

Using Eq. (4), it can be easily shown that for a given $T_{i g}$ and a thermally thin fuel bed with thickness $\delta$, given initial conditions $\left(T_{o}, q_{s}^{\prime \prime}\right)$ and known properties $\left(\rho, c_{p}\right)$,

$$
t_{i g} \sim M C
$$

The above equation has been used to correlate ignition time with moisture content for Douglas fir and lodgepole pine needles [4].

\section{Concluding Remarks}

A previously reported correlation between ignition time and moisture content of thermally thick fuel beds is derived using an alternative approach with the introduction of a firebrand heating efficiency. The approach is also extended to a thermally thin fuel bed. Analogous to 
the use of spray cooling efficiency and dimensional analysis to correlate heat transfer characteristics in spray cooling of hot surfaces, the idea of using a firebrand heating efficiency of a forest fuel bed coupled with dimensional analysis may offer an effective way to correlate prospective experimental ignition data as function of fuel properties, fuel configuration, firebrand shower characteristics, and wind.

\section{References}

[1] Yin P, Liu N, Chen H, Lozano, JS, Shan Y (2014) New correlation between ignition time and moisture content for pine needles attacked by firebrands. Fire Technology 50(1):79-91. https://doi.org/10.1007/s10694-012-0272-y

[2] Carslaw HS, Jaeger JC (1959) Conduction of heat in solids (2 ${ }^{\text {nd }}$ ed). Oxford University Press, Oxford, UK.

[3] Bolle L, Moureau JC (1981) Spray cooling of hot surfaces. Multiphase Science and Technology (vol. 1), Hewitt GF, Delhaye JM, Zuber N (editors). Hemisphere Publishing Co., Washington DC.

[4] Jolly M, McAllister S, Finney M, Hadlow, A (2010) Time to ignition is influenced by both moisture content and soluble carbohydrates in live Douglas fir and lodgepole pine needles. In: Viegas DX (ed) Proceedings of the VI International Conference on Forest Fire Research, Coimbra, Portugal, 15-18 November 2010. Coimbra: University of Coimbra. 